\title{
Suplementação oral de L-carnitina associada ao treinamento físico e muscular respiratório na doença pulmonar obstrutiva crônica: estudo preliminar
}

\author{
Oral supplementation of $L$-carnitine combined with exercise and respiratory training \\ in patients with chronic obstructive pulmonary disease: preliminary study
}

Matheus Guedes Fernandes Silva', Carolina Pereira Fernandes², Tadeu Candido da Silva Santos³, Tatiane Lopes Patrocínio da Silva ${ }^{4}$

\begin{abstract}
RESUMO I Avaliar os efeitos da suplementação oral de L-carnitina associada ao treinamento físico e muscular respiratório na doença pulmonar obstrutiva crônica (DPOC). Participaram 14 voluntários com idade de 65 $\pm 10,4$ anos e diagnóstico clínico de DPOC moderado, classificados de acordo com a espirometria prévia. Os voluntários foram divididos em grupo treino esteira (GTE) e grupo treino muscular respiratório (GTMR). Realizaram o teste de caminhada de seis minutos (TC6'), teste de caminhada com carga progressiva (TCP), avaliação nutricional do índice de massa corpórea (IMC), dose diária recomendada de L-carnitina, pressões inspiratórias (PImáx) e expiratórias máximas (PEmáx). Fizeram 30 min de caminhada em esteira, 3 vezes/semana por 10 semanas, e o GTMR realizou, ainda, 10 min de treinamento muscular inspiratório (Threshold ${ }^{\circledR} \mathrm{IMT}$ ) e 10 min de treinamento muscular expiratório (Threshold ${ }^{\circledR}$ PEP) à 50\% da PImáx e PEmáx ajustados semanalmente. Após 10 semanas, foram reavaliados. No TC6' pré e pós-programa de treinamento físico, as variáveis alteradas foram: distância percorrida (DP), frequência cardíaca (FC) final, pressão arterial sistólica (PAS) final, pressão arterial diastólica (PAD) final e Borg final no GTMR, no GTE as variáveis alteradas foram FC repouso, FC final, PAS final, Borg repouso e DP. Comparando os grupos no TC6, o GTE apresentou FC final, PAD final e Borg final maiores do que o GTMR na reavaliação; já no TCP, a FC final, PAS final, Borg final foram maiores no GTE, e DP foi maior no GTMR. Na avaliação respiratória, a PEmáx foi maior no GTMR na reavaliação. O treino aeróbio e suplementação de L-carnitina na DPOC otimizou a performance, a capacidade física e a tolerância ao esforço.
\end{abstract}

Descritores I carnitina; exercício; doença pulmonar obstrutiva crônica.

\begin{abstract}
I To evaluate the effects of oral supplementation of L-carnitine associated with physical and respiratory muscles training in chronic obstructive pulmonary disease (COPD). Participated 14 COPD volunteers (65 \pm 10.4 years), divided in group training mat (GTM) and respiratory muscle training group (RMTG). Passed by the six minute walk test (6MWT) and shuttle walk test (SWT), nutritional assessment of body mass index (BMI), dose recommended daily L-carnitine and evaluation of the inspiratory muscle training (IMT) and expiratory muscle training (EMT). They made 30 min walk on a treadmill 3 times/week for 10 weeks, and the RMTG also carried out 10 min with inspiratory muscle training (Threshold ${ }^{\circledR} \mathrm{IMT}$ ) and 10 min with expiratory muscle training (Threshold ${ }^{\circledR}$ PEP) with $50 \%$ of the MIP and MEP adjusted weekly. After 10 weeks, the volunteers were reevaluated. In 6MWT pre and post physical training programs, the variables changed were distance travelled (DT), final cardiac frequency (FCF), final systolic blood pressure (FSBP), diastolic blood pressure (DBP) and final Borg in RMTG. At GTM the variables changed were initial CF, final CF, SBP final, initial Borg and DT. Comparing the groups, we showed that in 6MWT, GTM presented final CF, final DBP and final Borg higher than RMTG in reevaluation. In shuttle walk test, the final SBP and final Borg were higher in GTM, and DT was higher in RMTG. In respiratory evaluation, the MEP was higher in RMTG in reevaluation. The aerobic training and L-carnitine supplementation in COPD patients presented performance optimization, improvement in physical capacity and greater exercise tolerance.
\end{abstract}

Keywords I carnitine; exercise; pulmonary disease, chronic obstructive.

Estudo desenvolvido no Departamento de Fisioterapia da Universidade de Taubaté (UNITAU) - Taubaté (SP), Brasil.

${ }^{1}$ Graduado em Fisioterapia pela UNITAU - Taubaté (SP), Brasil; Especialista em Fisiologia do Exercício pela Universidade Federal de São Paulo (UNIFESP) - São Paulo (SP), Brasil e em Fisiologia do Exercício e Treinamento Resistido na saúde na doença e no envelhecimento pela Universidade de São Paulo (USP) - São Paulo (SP), Brasil.

${ }^{2}$ Graduada em Nutrição pela Universidade de Mogi das Cruzes (UMC) - Mogi das Cruzes (SP), Brasil; Especialista em Saúde Pública pela UNITAU - Taubaté (SP), Brasil.

${ }^{3}$ Graduado em Fisioterapia pela UNITAU - Taubaté (SP), Brasil.

${ }^{4}$ Mestre em Fisioterapia pela Universidade Federal de São Carlos (UFSCar) - São Carlos (SP), Brasil; Docente do Departamento de Fisioterapia da UNITAU - Taubaté (SP), Brasil.

Endereço para correspondência: Matheus Guedes Fernandes Silva - Rua Avenca, 161 - Jardim Samambaia - CEP: 11680-000 - Ubatuba (SP), Brasil - E-mail: matheusgfs@bol.com.br Apresentação: mar. 2012 - Aceito para publicação: ago. 2012 - Fonte de financiamento: nenhuma - Conflito de interesse: nada a declarar - Parecer de aprovação no Comitê de Ética no 132/11. 


\section{INTRODUÇÃO}

A doença pulmonar obstrutiva crônica (DPOC) é uma enfermidade respiratória prevenível e tratável, caracterizada pela obstrução crônica do fluxo aéreo, não totalmente reversível. A obstrução do fluxo aéreo está associada a uma resposta inflamatória anormal dos pulmões a partículas ou gases tóxicos, causada pelo tabagismo. Embora a DPOC comprometa os pulmões, ela também produz consequências sistêmicas significativas ${ }^{1}$.

Associada ao aumento da demanda ventilatória, a disfunção muscular esquelética contribui para a intolerância ao exercício físico; os pacientes apresentam sarcopenia adicional ao processo de envelhecimento normal e alterações adaptativas, como redução da capilaridade e do número de enzimas oxidativas, reduzindo a capacidade aeróbia ${ }^{2}$. Kunikoshita et al. ${ }^{3}$, mostram em seu estudo expressiva redução na tolerância ao esforço associada à redução nas pressões respiratórias máximas em indivíduos DPOC.

Estudos de medicina esportiva têm demonstrado o efeito ergogênico da L-carnitina na melhora da performance, através da redução das taxas de depleção do glicogênio muscular e aumento da resistência à fadiga. A L-carnitina associada ao treinamento físico em DPOC por 6 semanas com uma dosagem de $2 \mathrm{~g} / \mathrm{dia}$, melhorou as taxas cardíacas no grupo suplementado quando comparados aos não suplementados. Ainda, o grupo que combinou L-carnitina com treinamento físico aumentou a distância percorrida no teste de caminhada de seis minutos (TC6') ${ }^{4}$.

A prática de exercícios físicos associada a suplementação de L-carnitina exerce ação sobre o metabolismo energético, melhora a capacidade de endurance, e causa alterações fenotípicas nas fibras musculares, quando combinado com o treinamento ${ }^{5}$.

A carnitina (3-hidroxi-4-N-trimetilamino-butirato) é uma amina quaternária com função fundamental na geração de energia pela célula, que facilita a oxidação da glicose e dos ácidos graxos para produzir adenosina trifosfato (ATP). O aumento do fluxo de substratos no Ciclo de Krebs poderia resultar em produção e utilização mais efetivas do oxigênio e melhora na capacidade de exercício. Portanto, as síndrome musculares caracterizadas por deficiência de L-carnitina são associadas a perdas funcionais, demonstrando a importância da L-carnitina na função muscular e na otimização da performance $e^{6,7}$.

Visto que a DPOC resulta em uma série de alterações fisiológicas e sistêmicas, este estudo teve como objetivo avaliar os efeitos da suplementação oral de L-carnitina associada ao treinamento físico e muscular respiratório na DPOC.

\section{MATERIAIS E MÉTODOS}

Foram incluídos neste estudo 14 pacientes com diagnóstico clínico de DPOC, estáveis, com idade superior a 50 anos e sedentários, classificados de acordo com a espirometria prévia, sendo que a seleção da amostra foi por conveniência. Este estudo foi desenvolvido na Unidade de Fisioterapia Respiratória da Universidade de Taubaté (UNITAU), após aprovação pelo Comitê de Ética em pesquisa humana da nossa instituição sob o parecer $n^{\circ} 132 / 11$. A amostra foi dividida aleatoriamente em dois grupos: Grupo treino esteira (GTE, $n=7)$, que recebeu suplementação de $2 \mathrm{~g} /$ dia de L-carnitina, ingerida de forma fracionada em duas doses diárias (manhã e tarde), com intervalo médio de $8 \mathrm{~h}$, sendo um deles $1 \mathrm{~h}$ antes da prática de exercícios físicos aeróbicos, e Grupo treino muscular respiratório (GTMR), que também recebeu suplementação de $2 \mathrm{~g} /$ dia de L-carnitina e realizou o mesmo treinamento em esteira, acrescido do treino muscular respiratório. A L-carnitina foi administrada em flaconetes contendo $1 \mathrm{~g}^{4}$.

\section{Avaliação clínica e da capacidade física}

Para avaliação da altura e peso, todos os pacientes foram pesados em uma balança de Impedância Bioelétrica (IB) da marca Tania 300, com roupas de banho e descalços, no período da manhã.

O TC6' foi realizado em um corredor plano de $30 \mathrm{~m}$ de comprimento; os pacientes foram monitorados durante todo o teste através de um oxímetro de pulso portátil (Nonim 8500A, Plymouth, Mn, USA), e foram questionados quanto a dispneia, no início e no final do teste, através da escala de percepção de esforço de Borg ${ }^{1,2}$.

O teste de caminhada com carga progressiva (TCP) foi realizado em um corredor plano de $10 \mathrm{~m}$ de comprimento e intensidade determinada por sinal sonoro padrão ${ }^{8}$. Os voluntários realizaram o TC6' e o TCP em dias e horas marcadas, com prévia instrução de como seriam realizados os testes, com roupas e calçados adequados, pelo menos uma hora após a última refeição ${ }^{8}$.

Visando evitar a interferência do aprendizado nos testes, e procurando garantir maior fidedignidade aos resultados, foram realizados dois TC6' e dois TCP em 
dias alternados, com 30 min de repouso entre os testes, sendo que o maior valor foi utilizado para análise estatística dos dados. O examinador orientava e incentivava os pacientes no início e no decorrer dos testes a caminhar o mais rápido possível, sendo que o encorajamento foi padronizado ${ }^{8}$. Os valores de saturação da oxiemoglobina $(\mathrm{SaO} 2)$ foram monitorados durante os testes, bem como a escala de Borg ${ }^{1,2}$.

\section{Avaliação muscular respiratória}

A força muscular respiratória foi avaliada através da medida das pressões inspiratórias (PImáx) e expiratórias máximas (PEmáx), a partir do volume residual e capacidade pulmonar total, respectivamente, com um manuvacuômetro escalonado em $-300 \mathrm{a}+300 \mathrm{cmH}_{2} 0$, de acordo com a metodologia proposta por Black e Hyatt ${ }^{9}$. Os pacientes foram instruídos a realizar três esforços máximos durante, no mínimo, um segundo, contra uma via ocluída com um pequeno orifício de escape para evitar que os pacientes mantivessem a glote aberta, evitando a ação dos músculos da parede da boca. A manobra foi realizada por três vezes com uso um clipe nasal. Para efeito de análise estatística foi considerado o maior valor alcançado.

\section{Programas de treinamento físico}

Os pacientes do GTE e do GTMR foram submetidos a um programa de treinamento físico (TF) que consistiu de 30 min de caminhada em esteira rolante realizado 3 vezes por semana, em dias não consecutivos, por 10 semanas com intensidade de treinamento em 40-50\% da frequência cardíaca (FC) máxima. Além disso, os pacientes foram orientados a realizar alongamentos de membros superiores e membros inferiores, antes de iniciar a caminhada. Este programa de treinamento teve duração de $1 \mathrm{~h}$. Cada sessão de TF, quando necessário, era precedido de inaloterapia e higiene brônquica. Além disso, o GTMR realizou 10 min de treinamento muscular inspiratório (Threshold ${ }^{\circledR}$ IMT) e 10 min de treinamento muscular expiratório (Threshold ${ }^{\circledR}$ PEP), da Respironics, respectivamente, com 50\% da PImáx e PEmáx a cada sessão, reavaliada semanalmente para reajuste da carga. Após 10 semanas, os voluntários foram reavaliados da mesma forma.

Para análise de distribuição dos dados, utilizou-se o teste de normalidade Shapiro-Wilks, após utilizou-se o teste de Wilcoxon nas comparações entre os momentos avaliação e reavaliação e o teste Mann-Whitney para comparações entre os grupos, pois a amostra não apresentou distribuição normal, o nível de significância, adotado foi de $\mathrm{p} \leq 0,05$.

\section{RESULTADOS}

Como resultados do TC6' antes e após os programas de treinamento físico, encontrou-se alterações significativas para as variáveis distância percorrida (DP), FC final, pressão arterial sistólica (PAS) final, pressão arterial diastólica (PAD) final e Borg final no GTMR; no GTE, as variáveis alteradas foram $\mathrm{FC}$ repouso, FC final, PAS final, Borg repouso e DP (Tabela 1).

Tabela 1. Comparação intra e entre os grupos entre as variáveis do teste de caminhada de seis minutos, índice de massa corpórea e pressões respiratórias máximas antes e após o programa de treinamento físico

\begin{tabular}{|c|c|c|c|c|}
\hline & \multicolumn{2}{|c|}{ GTE } & \multicolumn{2}{|c|}{ GTMR } \\
\hline & Antes & Após & Antes & Após \\
\hline $\mathrm{DP}(\mathrm{m})$ & $327,14 \pm 44,71$ & $345,57 \pm 62,68^{*}$ & $461,00 \pm 39,27$ & $515,42 \pm 48,81^{*}$ \\
\hline FC repouso (bpm) & $97,28 \pm 15,49$ & $80,85 \pm 3,67^{*}$ & $91,85 \pm 12,62$ & $76,71 \pm 3,45$ \\
\hline FC final (bpm) & $125,42 \pm 15,57$ & $108,85 \pm 12,57^{*}$ & $107,71 \pm 11,70$ & $86,14 \pm 3,67^{*}$ \\
\hline Borg repouso & $4,71 \pm 1,38$ & $4,42 \pm 1,27^{*}$ & $3,28 \pm 0,75$ & $0,71 \pm 0,75$ \\
\hline Borg Final & $6,14 \pm 1,46$ & $5,85 \pm 2,03$ & $5,42 \pm 0,78$ & $3,42 \pm 0,53^{*}$ \\
\hline PAS repouso (mmHg) & $132,85 \pm 17,99$ & $124,28 \pm 15,11$ & $122,85 \pm 9,51$ & $121,42 \pm 12,14$ \\
\hline PAD repouso $(\mathrm{mmHg})$ & $82,85 \pm 9,51$ & $81,42 \pm 8,99$ & $77,14 \pm 4,87$ & $74,28 \pm 5,34$ \\
\hline PAS final $(\mathrm{mmHg})$ & $141,42 \pm 15,73$ & $132,85 \pm 14,96 *$ & $125,71 \pm 9,75$ & $124,28 \pm 12,72^{*}$ \\
\hline PAD final $(\mathrm{mmHg})$ & $94,28 \pm 5,34$ & $94,28 \pm 7,86$ & $88,57 \pm 6,90$ & $80,0 \pm 5,77^{*}$ \\
\hline IMC ( $\left(\mathrm{kg} / \mathrm{m}^{2}\right)$ & $25,62 \pm 2,11$ & $26,02 \pm 1,83$ & $26,03 \pm 1,83$ & $26,30 \pm 1,64^{*}$ \\
\hline PImáx $\left(\mathrm{cmH}_{2} \mathrm{O}\right)$ & $86,28 \pm 2,13$ & $86,57 \pm 5,65$ & $78,42 \pm 10,08$ & $82,42 \pm 4,03^{*}$ \\
\hline PEmáx $\left(\mathrm{cmH}_{2} \mathrm{O}\right)$ & $71,71 \pm 12,98$ & $73,42 \pm 10,69^{*}$ & $82,57 \pm 10,24$ & $94,85 \pm 11,83^{*}$ \\
\hline
\end{tabular}

GTE: grupo treino esteira; GTMR: grupo treino muscular respiratório; DP: distância percorrida; FC: frequência cardíaca; Borg: pontuação na escala de Borg; PAS: pressão arterial sistólica; PAD: pressão arterial diastólica; IMC: índice de massa corpórea; PImáx: pressão inspiratória máxima; PEmáx: pressão expiratória máxima; p $\leq 0,05$

*Significativo para teste Wilcoxon e "Significativo para teste Mann- Whitney 
Como resultados do TCP antes e após os programas de treinamento físico, encontraram-se alterações significativas para DP, FC final, PAS final e Borg repouso no GTMR; no GTE, as variáveis alteradas foram FC final, Borg repouso, Borg final e DP (Tabela 2).

$\mathrm{Na}$ comparação entre os grupos, observou-se que no TC6', o GTE apresentou FC final, PAD final e Borg final maiores que o GTMR no momento da reavaliação; já, no TCP, a FC final, PAS final e Borg final foram maiores no GTE e DP maior no GTMR. Em relação à avaliação respiratória, a PEmáx foi maior na reavaliação no GTMR que realizou treinamento específico para este fim (Tabela1).

\section{DISCUSSÃO}

A DPOC é considerada importante causa de morbidade e mortalidade no mundo. Caracteriza-se por obstrução parcialmente reversível da via aérea e resposta inflamatória pulmonar, cursa com alterações nutricionais e da musculatura esquelética ${ }^{10}$.

A fisiopatologia da DPOC está relacionada à limitação do fluxo expiratório em resposta a redução do recuo elástico e aumento da resistência ao fluxo aéreo, tendo como principal sintoma a dispneia, que representa o fator primário da limitação ao exercício e resultará em disfunção muscular esquelética ${ }^{11}$.

Estudos com técnica de biópsia muscular na DPOC demonstraram alterações da atividade enzimática e metabólica indicando importante redução da capacidade oxidativa e glicolítica, além de acúmulo excessivo de fosfato inorgânico e acidose prematura em resposta á produção de lactato em musculatura periférica dos pacientes avaliados ${ }^{11,12}$. O conjunto desses fatores reduz a capacidade de exercício na DPOC, comprovando que a disfunção muscular esquelética relaciona-se com intolerância ao exercício nestes pacientes ${ }^{12,13}$.

O exercício aeróbico é primordial nos programas de reabilitação pulmonar, por aumentar a tolerância ao exercício e resultar em melhora do condicionamento cardiorrespiratório ${ }^{14,15}$. A suplementação com carnitina reduz os níveis de depleção do glicogênio muscular, aumenta a resistência à fadiga, a reposição da carnitina perdida durante o treinamento, e aumenta a oxidação dos ácidos graxos musculares, demonstrando eficiência na melhora da performance durante o exercício ${ }^{7}$. Esse fato foi comprovado em nosso estudo, uma vez que os pacientes com DPOC suplementados com carnitina aumentaram a capacidade física submáxima, observados pela análise dos dados obtidos no TC6' pré- e pós-programa de treinamento físico.

No TC6', os pacientes apresentaram aumento da distância percorrida, tanto no GTE, quanto no GTMR, o que nos leva a concluir que houve melhora da tolerância aos esforços submáximos dos voluntários após intervenção. A frequência cardíaca de repouso foi menor na reavaliação no GTE, já a frequência cardíaca final e a pressão arterial sistólica final foram menores para ambos os grupos; a pressão arterial diástólica final foi menor apenas para o GTMR. As reduções nas pressões e frequências cardíacas observadas neste estudo sugerem melhora do controle autonômico cardíaco, redução da atividade simpática e adaptação ao treinamento. Chalela e Moffa ${ }^{16}$ semelhantemente afirmam que a FC tem relação linear com a intensidade de exercício, com redução rápida devido ao retorno da atividade vagal com consequente redução da atividade simpática.

Tabela 2. Comparação intra e entre os grupos entre as variáveis do teste de caminhada com carga progressiva antes e após o programa de treinamento físico

\begin{tabular}{lcccc} 
& & GTE & & \multicolumn{2}{c}{ GTMR } \\
\cline { 2 - 5 } DP $(\mathrm{m})$ & Antes & Após & Antes & Após \\
FC repouso (bpm) & $194,85 \pm 22,06$ & $217,71 \pm 27,54^{*}$ & $282,71 \pm 19,42$ & $306,42 \pm 14,75^{*}$ \\
FC final (bpm) & $107,28 \pm 9,39$ & $93,28 \pm 5,55$ & $97,14 \pm 8,47$ & $86,0 \pm 4,76^{*}$ \\
Borg repouso & $123 \pm 11,88$ & $110 \pm 8,62^{*}$ & $106,71 \pm 7,95$ & $95,57 \pm 4,35^{*}$ \\
Borg final & $4,71 \pm 1,11$ & $4,71 \pm 1,49^{*}$ & $2,85 \pm 1,34$ & $1,57 \pm 0,53^{*}$ \\
PAS repouso (mmHg) & $8,14 \pm 0,89$ & $6,0 \pm 1,15^{*}$ & $5,0 \pm 0,57$ & $3,57 \pm 0,53^{*}$ \\
PAD repouso $(\mathrm{mmHg})$ & $135,71 \pm 9,75$ & $128,57 \pm 8,99$ & $128,57 \pm 3,77$ & $122,85 \pm 4,87$ \\
PAS final (mmHg) & $91,42 \pm 6,90$ & $90,0 \pm 5,77$ & $85,71 \pm 5,34$ & $81,42 \pm 3,77$ \\
PAD final $(\mathrm{mmHg})$ & $141,42 \pm 13,45$ & $137,14 \pm 7,55$ & $131,42 \pm 130,52$ & $130 \pm 8,16^{*}$ \\
\end{tabular}

GTE: grupo treino esteira; GTMR: grupo treino muscular respiratório; DP: distância percorrida; FC: frequência cardíaca; Borg: pontuação na escala de Borg; PAS: pressão arterial sistólica; PAD: pressão arterial diastólica; IMC: índice de massa corpórea; PImáx: pressão inspiratória máxima; PEmáx: pressão expiratória máxima; p $\leq 0,05$

*Significativo para teste Wilcoxon e "Significativo para teste Mann- Whitney 
$\mathrm{Na}$ percepção de esforço avaliada pela escala de Borg, os valores de repouso e ao final do teste foram menores na reavaliação para os dois grupo, o que pode-se correlacionar com a maior distância percorrida no TC6, pelos pacientes dos dois grupos que foram capazes de percorrer maiores distâncias com sensação subjetiva de esforço menor.

O índice de massa corpórea (IMC) foi maior na reavaliação apenas no GTMR, sem alteração nos pacientes do GTE, tal fato pode ter ocorrido devido à baixa variabilidade dos valores desse índice entre os dois grupos analisados. Coelho et al. ${ }^{17}$ não observaram alterações na composição corporal de indivíduos treinados por 30 dias, suplementados com L-carnitina. Alguns autores afirmam haver uma relação entre o baixo IMC, o comprometimento muscular periférico e a baixa capacidade de exercício em indivíduos com DPOC ${ }^{18,19}$. Hart et al. ${ }^{20}$ afirmaram que o IMC é importante preditor da força diafragmática, já que o fator nutricional tem efeito sobre a força dessa musculatura. $\mathrm{O}$ aumento na resistência mecânica da parede torácica causa maior resistência elástica, dificulta a expansão do tórax durante a inspiração, e compromete a complacência total do sistema respiratório ${ }^{21}$; portanto, podemos correlacionar em algum grau a dificuldade respiratória dos voluntários com o baixo IMC apresentado por eles.

A força muscular respiratória medida pelas PImáx e PEmáx tem sido estudadas nas últimas décadas como uma técnica eficaz para estimar a condição respiratória das pessoas, e possível intervenção ${ }^{22}$.

Os músculos respiratórios são fundamentais na manutenção da mecânica respiratória e, em condições fisiopatológicas, sua força encontra-se alterada resultando em diminuição das pressões respiratórias $^{23}$. A hiperinsuflação dinâmica presente na DPOC também compromete a performance muscular respiratória, modificando a conformação geométrica das fibras musculares e reduzindo a curvatura diafragmática ${ }^{24,25}$.

Neste estudo, ao avaliarmos as pressões respiratórias máximas, observarmos que o incremento nos valores da PImáx só foi relevante no grupo que realizou treinamento específico para este fim, já na avaliação da PEmáx houve incremento da PEmáx tanto no GTE, quanto no GTMR, fato relevante e que associado a outros fatores já citados pode correlacionar-se com a melhora da capacidade física observada nos voluntários.
No TCP, concluímos que houve melhora na capacidade máxima de exercício, pois os voluntários apresentaram aumento na distância percorrida tanto no GTE, quanto no GTMR, evidenciando o benefício entre suplementação de carnitina e treino aeróbico na otimização da performance. A FC de repouso foi menor na reavaliação para o GTMR, já a FC final foi menor na reavaliação para ambos os grupos, o que nos mostra que também durante a realização do TCP houve boa adaptação do sistema cardiorrespiratório ao estresse gerado pelo exercício.

A PAS final foi menor na reavaliação no GTMR, esse menor índice pressórico pode correlacionar-se a uma resposta adaptativa do sistema cardiovascular ao treinamento, sendo uma resposta esperada após algumas semanas de treinamento, visto que o mesmo é capaz de reduzir pré- e pós-carga cardíaca, ou seja, o coração consegue 'trabalhar' menos pra uma mesma intensidade de esforço quando comparado ao condicionamento cardiovascular prévio à reabilitação.

O Borg de repouso e o Borg final reduziram-se tanto no GTE, quanto no GTMR. Podemos correlacionar este fato a maior distância percorrida pelos voluntários dos dois grupos, pois com uma menor sensação subjetiva de esforço, os voluntários foram capazes de percorrer uma maior distância (caminhar mais) e sentir menos cansaço.

Portanto, a interação entre treino aeróbio e suplementação de L-carnitina na DPOC mostrou-se benéfica, pois os pacientes apresentaram otimização da performance, melhora da capacidade física e funcional, além de maior tolerância ao esforço e incremento da força muscular respiratória no grupo que realizou treinamento específico para este fim.

\section{Limitação do estudo}

O tamanho reduzido da amostra pode ser considerado como limitação do presente estudo. Neste estudo, não foi possível atingir uma amostra maior devido à dificuldade de recrutamento de pacientes com DPOC em nosso centro de estudos e no encaminhamento deles para o nosso programa de reabilitação pulmonar. Como limitação do presente estudo, destacamos, ainda, a dificuldade de acesso para realizar a bioimpedância em nosso serviço e a ausência de um grupo controle de treinamento sem suplementação de L-carnitina, que pode limitar os resultados do presente estudo. 


\section{REFERÊNCIAS}

1. II Consenso Brasileiro sobre Doença Pulmonar Obstrutiva Crônica. J Bras Pneumol. 2004;30.

2. Borghi-Silva A, Sampaio LMM, Toledo A. Efeitos agudos da aplicação do BiPAP sobre a tolerância ao exercício físico em pacientes com doença pulmonar obstrutiva crônica. Rev Bras Fisioter. 2005;9(3):273-80.

3. Kunikoshita LN, Silva TLP, Silva PY, Costa D, Jamami M. Efeitos de três programas de fisioterapia respiratória (PFR) em portadores de DPOC. Rev Bras Fisioter. 2006;10(4):445-9

4. Villaça DS, Lerario MC, Simone DC, Neder JA. New treatments for chronic obstructive pulmonary disease using ergogenic aids. J Bras Pneumol. 2005:32(1):66-74.

5. Lee KJ, Lee SJ, Park H, Chá SY, Yoon SCH, Kim CK. Effect of the L-carnitine supplementation and aerobic training on FABPC content and B- HAD activity in human skeletal muscle. Eur J Appl Physiol. 2007;99(2):193-9.

6. Coelho FC, Mota FJ, Bragança E, Burini CR. Aplicações clínicas da suplementação de carnitina. Rev Nutr. 2005;18(5):651-9.

7. Eric PB. Carnitine and sports medicine: Use or Abuse? Ann N Y Acad Sci. 2004:1033:67-78.

8. Rosa FW, Camelier A, Mayer A, Jardim JR. Avaliação da capacidade de exercício em portadores de doença pulmonar obstrutiva crônica: comparação do teste de caminhada com carga progressiva com o teste de caminhada com acompanhamento. J Bras Pneumol. 2006;32(2):106-13.

9. Black LF, Hyatt RE. Maximal respiratory pressures: normal values and relationship to age and sex. Am Rev Res Dis. 1969;99(5):696-702.

10. Simon MK, Hass PA, Zimmermman LJ, Carpes FM. Índice prognóstico de mortalidade $B O D E$ e atividade física em doentes pulmonares obstrutivos crônicos. RBME. 2009:15:19-22.

11. O'Donnell DE. Hyperinflation, dyspnea, and exercise intolerance in chronic obstructive pulmonary disease. Proc Am Thorac Soc. 2006:3(2):180-4.

12. Serres I, Hayot M, Préfaut C, Mercier J. Skeletal muscle abnormalities in patients with COPD: contribution to exercise intolerance. Med Sci Sports Exerc. 1998;30(7):1019-27.

13. Aliverti A, Macklen P. The major limitation to exercise performance in COPD is lower limb muscle dysfunction. Eur J Appl Physiol. 2007;99:265-74.
14. Bernard S, Whittom F, Leblanc P, Jobin J, Belleau R, Chantal B, et al. Aerobic and strength training in patients with chronic obstructive pulmonary disease. Am J Respir Crit Care Med. 1999;159(3):896-901.

15. Clark CJ, Cochrane L, Mackay E. Low intensity peripheral muscle conditioning improves exercise tolerance and breathlessness in COPD. Eur Respir J. 1996;9(12):2590-6.

16. Chalela WA, Moffa PJ. Teste ergométrico. Cardiologia do exercício: do atleta ao cardiopata. Barueri: Manole; 2005

17. Coelho FC, Mota FJ, Ravagnani PCF, Burini CR. A suplementação de L- carnitina não promove alterações na taxa metabólica de repouso e na utilização dos substratos energéticos em indivíduos ativos. Arq Bras Endocrinol Metab. 2010;54(1):37-44.

18. Kobayashi A, Yoneda T, Yoshikawa M, Ikuno M, Takenaka H, Fukuoka A, et al. The relation of fat-free mass to maximum exercise performance in patients with chronic obstructive pulmonary disease. Springer New York. 2000;178(2):119-27.

19. Eid AA, Ionescu AA, Nixon L, Lewis-Jenkins V, Matthews SB, Griffiths TL, et al. Inflammatory response and body composition in chronic obstructive pulmonary disease. Am J Respir Crit Care Med. 2001; 164(8 Pt 1):1414-8.

20. Hart N, Tounian P, Clément A, Boulé M, Polkey MI, Lofaso F, et al. Nutritional status is an important predictor of diaphragm strength in young patients with cystic fibrosis. Am J Clin Nutr. 2004; 80(5):1201-6.

21. Wadström C, Muller-Suur R, Backman L. Influence of excessive weight Ioss on respiratory function. Eur J Surg. 1991;157(5):341-6.

22. Benício DCN, Gastaldi CA, Perecin CJ, Avena MK, Guimarães CR, Sologuren JJM, et al. Medidas espirométricas em pessoas eutróficas e obesas nas posições ortostática, sentada e deitada. RAMB. 2004:50:541-53.

23. Moreno AM, Catai AM, Teodori MR, Borges ALB, Cesar CM, Silva E. Efeito de um programa de alongamento muscular pelo método de reeducação postural global sobre a força muscular respiratória e a mobilidade toracoabdominal de homens jovens sedentários. J Bras Pneumol. 2007;33:679-86.

24. III Consenso Brasileiro de Ventilação Mecânica. J Bras Pneumol. 2007:33:111-8.

25. Silva AB, Di Lorenzo VA, Jamam M, Sampaio LM, Demonte A Cardello L, et al. Efeitos da suplementação oral de L-carnitina associada ao treinamento físico na tolerância ao exercício de pacientes com doença pulmonar obstrutiva crônica. J Pneumol. 2003;29(6):379-85. 